\title{
THE BURKILL APPROXIMATELY CONTINUOUS INTEGRAL
}

\author{
P. S. BULLEN
}

(Received 2 I December 1981)

Communicated by Gavin Brown

\begin{abstract}
This paper defines descriptive, Riemann, and constructive integrals equivalent to the approximately continuous integral of Burkill.
\end{abstract}

1980 Mathematics subject classification (Amer. Math. Soc.): 26 A 39.

\section{Introduction}

The simplest and most natural integral that integrates finite approximate derivatives is that of Burkill, [4]. However except for an important work of Tolstov, [25], it has not received much attention, in contrast to some fairly extensive investigations of other approximately continuous integrals; see Bullen, [3], for details and references. In this paper several alternative definitions of this Perron integral will be given; a descriptive integral, a totalization process, and a Riemann-like integral that has been suggested by Henstock, [6-8].

\section{The Burkill integral and its basic properties}

Definition 1. (a) Let $f:[a, b] \rightarrow \overrightarrow{\mathbf{R}}$; then $M$ is a major function of $f, M \in M_{f}^{\#}$, if and only if $M:[a, b] \rightarrow \mathbf{R}$ and:

(i) $M$ is approximately continuous, $M \in C_{a p}$;

[This work was done while the author was a visiting professor at the University of Melbourne.]

(c) 1983 Australian Mathematical Society 0263-6115/83 \$A2.00+0.00 
(ii) $M(a)=0$;

(iii) $l M_{a p}^{\prime}>-\infty$ n.e. (except on a countable set);

(iv) $l M_{a p}^{\prime} \geqslant f$ a.e.

(b) $m$ is a minor function of $f, m \in M_{\#, f}$, if and only if $-m \in M_{-f}^{\#}$.

(c) $F$ is $P_{a p}^{*}$-integrable, $f \in P_{a p}^{*}$ if and only if

$-\infty<\sup \left\{t ; t=m(b), m \in M_{\#, f}\right\}=\inf \left\{t ; t=M(b), M \in M_{f}^{\#}\right\}<\infty$,

when the common value will be written $\int_{a}^{f} f$.

REMARKS. (1) In case of ambiguity we will talk about $P_{a p}^{*}$-major functions on $[a, b]$, and so on.

(2) Clearly if $f \in P_{a p}^{*}$ then $M_{f}^{\#} \neq \varnothing, M_{\#, f} \neq \varnothing$.

Lemma 2. (a) If $M \in M_{f}^{\#}$ then $M$ is measurable, $M \in l[A C G], M_{a p}^{\prime}$ exists, finite, a.e.

(b) If $M \in M_{f}^{\#}$ and $m \in M_{\#, f}$ then $M-m$ is non-negative, increasing, continuous and differentiable a.e.

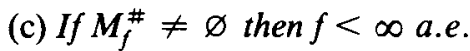

(d) If $f \in P_{a p}^{*}$ then $f$ is finite a.e.

Proof. (a) follows from results due to Ridder, [17, 18], while (b) follows from a result of Tolstov, [24], and O'Malley, [15], Sunouchi and Utagawa, [22]. (c), (d) are easy consequences of Definition 1.

REMARKS. (1) A function is $l[A C G]$ when $[a, b]$ is a countable union of closed sets on each of which it is lower absolutely continuous (see Ridder, [17-18]).

(2) The basic properties of the integral follow in the usual way; see, for instance, Burkill, [4]. In particular if $f \in P_{a p}^{*}$ then the $P_{a p}^{*}$-primitive, $F(x)=\int_{a}^{x} f$, $a \leqslant x \leqslant b$, is well-defined.

THeOREM 3. (a) If $f \in P_{a p}^{*}, M \in M_{f}^{\#}, m \in M_{\#, f}, F(x)=\int_{a}^{x} f$ then $M-F$ and $F-m$ are non-negative, increasing, continuous and differentiable a.e.

(b) If $f \in P_{a p}^{*}, F(x)=\int_{a}^{x} f$ then $F \in[A C G], F \in C_{a p}$ and $F_{a p}^{\prime}=$ fa.e.

(c) If $f \in P_{a p}^{*}$ then $f$ is measurable.

(d) If $F \in C_{a p}$ and (i) $F_{a p}^{\prime}(x)$ exists, finite, $x \notin E,|E|=0$, (ii) $u F_{a p}^{\prime}$ and $l F_{a p}^{\prime}$ are finite n.e., then if

$$
\begin{aligned}
f(x) & =F_{a p}^{\prime}(x), \quad x \notin E, \\
& =0, \quad x \in E,
\end{aligned}
$$

$f \in P_{a p}^{*}$ 
(e) The $P_{a p}^{*}$ - and the $D$-integrals are compatible.

(f) If $f \in P_{a p}^{*}[\alpha, \beta]$, for all $\alpha, \beta, a<\alpha<\beta<b$ and if

$$
\lim _{\substack{\alpha \rightarrow a \\ \beta \rightarrow b}} \int_{\alpha}^{\beta} f
$$

exists, with value I say, then $f \in P_{a p}^{*}[a, b]$ and $\int_{a}^{b} f=I$.

(g) Let $f \in P_{a p}^{*}, F(x)=\int_{a}^{x} f$ then for all $\lambda, 0<\lambda<1, P$ perfect, there exists $a$ closed portion, $Q$, of $P$, having, on $[a, b]$, closed contiguous intervals $\left[a_{n}, b_{n}\right]$, $n \in N$, such that for all $n \in N$ there exists an $E_{n} \subset\left[a_{n}, b_{n}\right]$, and an $M>0$, with $\left|E_{n}\right| \geqslant(1-\lambda)\left(b_{n}-a_{n}\right)$ and such that for all $x_{n} \in E_{n}, \Sigma_{n}\left|F\left(x_{n}\right)-F\left(a_{n}\right)\right|<M$ and $\Sigma_{n}\left|F\left(b_{n}\right)-F\left(x_{n}\right)\right|<M$.

(h) $D-P_{a p}^{*} \neq \varnothing$ and $P_{a p}^{*}-D \neq \varnothing$.

Proof. (b) is due to Kubota, [9]; (e) is in Kubota, [10]; (f) is a result of Grimshaw, [5]; (g) is due to Tolstov, [25]; the rest either follow easily from Lemma 2, or other parts of Theorem 3, or can be found in these references, or in Burkill, [4].

Definition 1 is not exactly that given in Burkill, [4], and the object of the next lemma is to show that the two definitions give equivalent integrals. Let Definition 1(a) be modified by replacing (iii) and (iv) by:

(iii) ${ }^{1} M_{a p}^{\prime}>-\infty$;

(iv) ${ }^{1} M_{a p}^{\prime} \geqslant f$;

and denote the resulting class of major functions by $M_{f}^{\# 1}$. Clearly $M_{f}^{\# 1} \subset M_{f}^{\#}$.

Lemma 4. For all $\varepsilon>0, M \in M_{f}^{\#}$ there exists $M^{1} \in M_{f}^{\# 1}$ such that

$$
M^{1}(b) \leqslant M(b)+\varepsilon .
$$

Proof. (a) Suppose Definition l(a) is modified by replacing (iii) by:

(iii) $^{2} l M_{a p}^{\prime}>-\infty$, and call the resulting class of major functions $M_{f}^{\# 2}$. We first prove the lemma with 1 replaced by 2 .

First suppose that the countable exceptional set in Definition 1(a)(iii) is the singleton $\{c\}, a<c<b$ (the cases $c=a, c=b$ can be discussed in a similar way).

Let $\varepsilon>0, M \in M_{f}^{\#}$ and let $A$ be a set of density 1 at $c$ on which $M$ is continuous; choose $a_{1}, b_{1}$ so that $a<a_{1}<c<b_{1}<b$ and the oscillation of $M$ on $A \cap\left[a_{1}, b_{1}\right]$ is less than $\varepsilon$. Define $\omega$ by

$$
\omega(x)=\sup \{t: t=|M(y)-M(c)|, y \in A,|y-c| \leqslant|x-c|\}
$$


and let $\chi$ be an increasing, differentiable function with $\chi(a)=0, \chi(b)=\varepsilon$, $\chi^{\prime}(c)=\infty$. Now define

$$
\begin{aligned}
& M^{2}(x)=M(x)+\chi(x), \quad a \leqslant x \leqslant a_{1}, \\
& =M(x)+\chi(x)+\omega\left(a_{1}\right)-\omega(x), \quad a_{1} \leqslant x \leqslant c, \\
& =M(x)+\chi(c)+\omega\left(a_{1}\right)+\omega(x), \quad c \leqslant x \leqslant b_{1}, \\
& =M(x)+\chi(x)+\omega\left(a_{1}\right)+\omega\left(b_{1}\right), \quad b_{1} \leqslant x \leqslant a ;
\end{aligned}
$$

then $M^{2} \in M_{f}^{\# 2}$ and (1) holds.

If we let $\Delta=M^{2}-M=\chi+\mu$ then the essential properties of $\mu$ are that it is increasing, continuous, $\mu(a)=0, \mu(b)<2 \varepsilon$, and on a set of $h$ having density 1 at the origin

$$
M(c+h)-M(c)+\mu(c+h)-\mu(c) \geqslant 0 .
$$

Now suppose that the countable exceptional set in Definition 1(a)(iii) is $c_{n}$, $n \in N$, and for each $c_{n}$ define a $\Delta_{n}$, as $\Delta$ was defined above, but with $\varepsilon$ replaced by $\varepsilon 2^{n}$; then if $M^{2}=M+\Sigma_{n} \Delta_{n}, M \in M_{f}^{\# 2}$ and (1) holds.

(b) From Lemma 2(a) it follows that (iv), in the definition of $M_{f}^{\# 2}$, can be replaced by

(iv) ${ }^{2} M_{a p}^{\prime} \geqslant f$, a.e.,

without affecting the definition of the integral.

(c) From (b) given $\varepsilon>0, M \in \tilde{M}_{f}$ there exists $M^{2} \in M_{f}^{\# 2}$, satisfying (iv) ${ }^{2}$, such that (1) holds. Now let

$$
E=\left\{x ;\left(M^{2}\right)_{a p}^{\prime}(x)<F(x), \text { or }\left(M^{2}\right)_{a p}^{\prime}(x) \text { does not exist }\right\} ;
$$

then $|E|=0$. If then $T \in G_{\delta}, E \subset T,|T|=0$ there exists a function $g:[a, b] \rightarrow \mathbf{R}$ such that (i) $g \in A C$, (ii) $g$ is increasing, (iii) $g$ is differentiable, (iv) $g^{\prime}(x)=\infty$, $x \in T$, (v) $g^{\prime}(x) \neq \infty, x \notin T$, (vi) $g(a)=0$, (vii) $g(b) \leqslant \varepsilon$; Zahorski, [27], Tolstov, [26]. Now if $M^{1}=M^{2}+g$ then $M^{1} \in M_{f}^{\# 1}$ and (1) holds.

Remarks. (1) The basic ideas for this lemma can be found in Aleksandrov, [1], Bosanquet, [2] and Grimshaw, [5].

(2) Burkill used the class $M_{f}^{\# 2}$ to define his integral. It should also be remarked that there would be no loss in generality in assuming, in Definition 1, that $f$ is finite, for in any case integrable functions are finite a.e. and if $f_{1}=f_{2}$ a.e. then $f_{1}$ and $f_{2}$ are either both not integrable, or both integrable with the same integral.

Following Henstock, [6], a definition of Ward type can be given. Suppose Definition 1(a) is modified by replacing (iii) and (iv) by:

(iii) $^{W}$ For all $x, a \leqslant x \leqslant b$, there exists a set $E_{x}$ of density 1 at $x$ such that $M(u)-M(v) \geqslant f(x)(u-v), u \leqslant x \leqslant v, u, v \in E_{x}$, and call the resulting class of major functions $W M_{f}^{\#}$. 
As in Henstock it follows that the integral defined this way, the $W P_{a p}^{*}$-integral, is equivalent to the one obtained from Definition 1 in which all the exceptional sets (Definition 1(a), (iii), (iv)) are empty, and the function $f$ finite. Hence from the above discussion this integral of Ward type is equivalent to the $P_{a p}^{*}$-integral.

A different sort of variant of Definition 1 has been given by Sunouchi and Utagawa, [22]. In Definition 1(a) replace (1), (iii) and (iv) by:

$S U$-(i) $M$ is measurable;

$S U$-(iii) $l M_{a p}^{\prime}>-\infty$ (that is, (iii) ${ }^{2}$ );

$S U$-(iv) $l M_{a p}^{\prime} \geqslant f$.

REMARK. The idea for this generalization is due to Saks, [20], who did the same for the classical Perron integral; he showed that the apparently more general integral was in fact equivalent to the original definition. We shall do the same in the present situation; until then we will call the integral defined this way the $S U$ - $P_{a p}^{*}$-integral. In their work, Sunouchi and Utagawa assumed $f$ to be measurable but this is unnecessary as this property of integrable $f$ can be proved (Theorem 3(c)).

\section{A Riemann definition}

A Riemann definition of an integral equivalent to the Burkill integral is suggested in Henstock, $[7,8]$, but no details are given.

Definition 1. (a) A collection, $\Delta$, of closed sub-intervals of $[a, b]$ is an approximate full cover of $[a, b]$, an AFC, if and only if for all $x, a \leqslant x \leqslant b$, there exists a measurable set $D_{x}, x \in D_{x}$, of density 1 at $x$, such that if $\alpha \leqslant x \leqslant \beta$, $\alpha, \beta \in D_{x}$, then $[\alpha, \beta] \in \Delta$.

(b) If $\Delta$ is an AFC of $[a, b]$ then a $\Delta$-partition of $[a, b]$ is a $\left\{a_{0}, \ldots, a_{n}\right.$; $\left.x_{1}, \ldots, x_{n}\right\}$, where $a=a_{0}<\cdots<a_{n}=b, a_{i-1} \leqslant x_{i} \leqslant a_{i}, a_{i-1}, a_{i} \in D_{x_{i}}, 1 \leqslant i \leqslant$ $n$.

Lemma 2. If $\Delta$ is an $A F C$ of $[a, b]$ and $a \leqslant \alpha<\beta \leqslant b$ then there exists $a$ $\Delta$-partition of $[\alpha, \beta]$.

Proof. This is a result of Thomson, [23].

Definition 3. (a) If $f:[a, b] \rightarrow r \mathbf{R}$ then $f$ is $R_{a p}^{*}$-integrable, $f \in R_{a p}^{*}$, if and only if there exists $I$ such that for all $\varepsilon>0$ there exists AFC, $\Delta$, of $[a, b]$, such 
that for all $\Delta$-partitions $\left\{a_{0}, \ldots, a_{n} ; x_{1}, \ldots, x_{n}\right\}$ of $[a, b]$ we have that

$$
\left|I-\sum_{i=1}^{n} f\left(x_{i}\right)\left(a_{i}-a_{i_{1}}\right)\right|<\varepsilon
$$

and then $\int_{a}^{b} f=I$.

(b) If $f:[a, b] \rightarrow \mathbf{R}$ then $f$ is $V R_{a p}^{*}$-integrable, $f \in V R_{a p}^{*}$, if and only if there exists $F:[a, b] \rightarrow \mathbf{R}$ such that for all $\varepsilon>0$ there exists AFC, $\Delta$, of $[a, b]$, and a non-decreasing $\phi:[a, b] \rightarrow \mathbf{R}$, with $\phi(b)-\phi(a)<\varepsilon$, such that for all $u, v$, $u \leqslant x \leqslant v, u, v \in D_{x}$, we have

$$
|F(v)-F(u)-f(x)(v-u)| \leqslant \phi(v)-\phi(u),
$$

and then $\int_{a}^{b} f=F(b)-F(a)$.

REMARKs. (1) The $R_{a p}^{*}$-integral is an example of what Henstock, [6], calls a Riemann complete integral, while the $V R_{a p}^{*}$-integral is an example of what he calls a variational integral; see also Kubota, [13, 14].

(2) The basic properties of these integrals follow in the standard manner; in particular we can talk of the $R_{a p}^{*}$-primitive, and the function $F$ in (b) above (unique by Theorem 5 below) is the $V R_{a p}^{*}$-primitive.

(3) It is also easily seen that if $R^{*}$ denotes Henstock's Riemann complete integral, that is equivalent to the classical Perron integral, then $R^{*} \subsetneq R_{a p}^{*}$.

LEMMA 4. (a) $f \in R_{a p}^{*}$, with primitive $F$, if and only if for all $\varepsilon>0$ there exists $A F C, \Delta$, of $[a, b]$, such that for all $\Delta$-partitions $\left\{a_{0}, \ldots, a_{n} ; x_{1}, \ldots, x_{n}\right\}$ of $[a, b]$ we have that

$$
\sum_{i=1}^{n}\left|F\left(a_{i}\right)-F\left(a_{i-1}\right)-f\left(x_{i}\right)\left(a_{i}-a_{i-1}\right)\right|<\varepsilon .
$$

(b) There is no loss in generality if, in Definition 3(b), it is assumed that $\phi \in C_{a p}$.

Proof. The proofs are similar to those for the $R^{*}$-integral; Henstock, 77 ; page $33,41]$.

TheORem 5. $f \in R_{a p}^{*}$ if and only if $f \in V R_{a p}^{*}$, and then the integrals are equal.

Proof. The proof follows that in Henstock [7; page 40]; see also Kubota [14].

REMARK. If $E \subset[a, b],|E|=0$ and if

$$
\begin{aligned}
1_{E}(x) & =1, \quad \\
& =0, \quad x \in E,
\end{aligned}
$$


then $1_{E} \in R^{*}$ and $\int_{a}^{b} 1_{E}=0$ : This can be used, in the usual way, to extend Definition 3 to functions that are finite a.e.

Let $\Delta$ be an AFC of $[a, b], \pi=\left\{a_{0}, \ldots, a_{n} ; x_{1}, \ldots, x_{n}\right\}$ a $\Delta$-partition of $[a, b]$; following Pfeffer, [16], we will write

$$
\begin{gathered}
S(f ; a, b ; \pi)=\sum_{i=1}^{n s} f\left(x_{i}\right)\left(a_{i}-a_{i-1}\right), \\
u S(f ; a, b ; \Delta)=\sup _{\pi} S(f ; a, b ; \pi), \\
u S(f ; a, b)=\inf _{\Delta} u S(f ; a, b ; \Delta),
\end{gathered}
$$

with analogous definitions of $l S(f ; a, b ; \Delta)$ and $l S(f ; a, b)$.

THEOREM 6. $f \in R_{a p}^{*}$ if and only if $-\infty<l S(f ; a, b)=u S(f ; a, b)<\infty$.

Proof. The proof follows that in Pfeffer, [16].

We can now show that the $P_{a p}^{*}$ and $S U-P_{a p}^{*}$-integrals are equivalent, and are equivalent to the $R_{a p}^{*}$-integral.

Lemma 7. If $A=\inf \left\{t ; t=M(b), M \in S U-M_{f}^{\#}\right\}$ then $A \geqslant u S(f ; a, b)$.

Proof. Let us assume $A<u S(f ; a, b)$, when there exists $M \in S U-M_{f}^{\#}$ such that $M(b)<u S(f ; a, b)$.

Given $\varepsilon>0, x, a \leqslant x \leqslant b$, set $E_{x}$ of density 1 at $x$ such that if $u, v \in E_{x}$ then

$$
M(v)-M(u) \geqslant(f(x)-\varepsilon)(v-u) .
$$

This defines an AFC, $\Delta$, of $[a, b]$; let $\pi=\left\{a_{0}, \ldots, a_{n} ; x, \ldots, x_{n}\right\}$ be a $\Delta$-partition of $[a, b]$ and consider

$$
S(f, a, b ; \pi)=\sum_{i=1}^{n} f\left(x_{i}\right)\left(a_{i}-a_{i-1}\right) \leqslant M(b)+\varepsilon(b-u) ;
$$

or

$$
u S(f ; a, b) \leqslant M(b) .
$$

COROLlaRY 8. SU-P $P_{a p}^{*} \subset R_{a p}^{*}$.

Proof. Immediate from Lemma 7 and Theorem 6.

LEMMA 9. $V R_{a p}^{*} \subset P_{a p}^{*}$. 
Proof. Let $f \in V R_{a p}^{*}, F, \phi$ as given in Definition 3(b), $\phi \in C_{a p}$, by Lemma 4(b); consider

$$
\mathbf{M}=\mathbf{F}+\boldsymbol{\phi}, \quad \boldsymbol{m}=\mathbf{F}-\boldsymbol{\phi} .
$$

Then $M \in W M_{f}^{\#}, m \in W M_{\#, f}$ and so $f \in W P_{a p}^{*}$ and hence $f \in P_{a p}^{*}$.

COROLlaRY 10. (a) $P_{a p}^{*}=S U P_{a p}^{*}$. (b) $R_{a p}^{*}=P_{a p}^{*}$.

Proof. Immediate from Corollary 8, Lemma 9 and Theorem 5.

REMARK. The above method can be used to given an alternative proof of Sak's result for the classical Perron integral.

\section{A descriptive definition}

Definition 1. (a) $F \in A C_{a p}^{*}$ on a closed set $E, F \in A C_{a p}^{*}(E)$, if and only if (i) $F \in A C(E)$, (ii) for all $\lambda, 0<\lambda<1$, there exists, on each closed contiguous interval of $E,\left[a_{n}, b_{n}\right]$, a set $E_{n}^{\lambda}$, and an $M^{\lambda}>0,\left|E_{n}^{\lambda}\right|>(1-\lambda)\left(b_{n}-a_{n}\right)$ such that for all $x_{n} \in E_{n}^{\lambda}, \Sigma_{n \in N}\left|F\left(x_{n}\right)-F\left(a_{n}\right)\right|<M^{\lambda}$, and $\Sigma_{n \in N}\left|F\left(b_{n}\right)-F\left(x_{n}\right)\right|<$ $M^{\lambda}$.

(b) $F \in\left[A C G_{a p}^{*}\right]$ on $[a, b]$ if and only if $[a, b]=\cup_{n \in N} E_{n}, E_{n}$ closed and $F \in A C_{a p}^{*}\left(E_{n}\right), n \in N$.

RemarK. It follows from Solomon's lemma, [1], that Definition 1(b) can be rephrased as:

$F \in\left[A C G_{a p}^{*}\right]$ on $[a, b]$ if and only if for all $\lambda, 0<\lambda<1, P$ perfect, there exists a closed portion $Q$ of $P$, having on $[a, b]$ closed contiguous intervals $\left[a_{n}, b_{n}\right], n \in N$, such that for all $n \in N$ there exists $E_{n}^{\lambda} \subset$ $\left[a_{n}, b_{n}\right], M^{\lambda}>0,\left|E_{n}^{\lambda}\right|>(1-\lambda)\left(b_{n}-a_{n}\right)$ and such that for all $x_{n} \in E_{n}^{\lambda}$, $\Sigma_{n \in N}\left|F\left(x_{n}\right)-F\left(a_{n}\right)\right|<M^{\lambda}$ and $\Sigma_{n \in N}\left|F\left(b_{n}\right)-F\left(\lambda_{n}\right)\right|<M^{\lambda}$.

We will first obtain some alternative forms of Definition 1(a). Let us define for $F:[a, b] \rightarrow \mathbf{R}$ and $A \in[a, b]$

$$
\omega(F ; A)=\sup \{t ; t=|F(x)-F(y)|, x, y \in A\} .
$$


LEMMA 2. If $E$ is a bounded closed set, with extremities $a, b, a<b$, and closed contiguous intervals in $[a, b],\left[a_{n}, b_{n}\right], n>1$, then if $E_{n} \subset\left[a_{n}, b_{n}\right], a_{n}, b_{n} \in E_{n}$, $n \geqslant 1, E_{0}=E \cup \cup_{n \geqslant 1} E_{n}$,

$$
\omega\left(F ; E_{0}\right) \leqslant V(F ; E)+2 \sum_{n \geq 1} \omega\left(F ; E_{n}\right),
$$

where $V(F ; E)$ is the variation of $F$ on $E$.

(This is a slight generalization of a result in Saks, [1; page 231].)

LEMMA 3. If $f \in C_{a p}[a, b]$ then for all $\lambda, 0<\lambda<1$, there exists $E^{\lambda} \subset[a, b]$, $a, b \in E^{\lambda}$ such that $\left|E^{\lambda}\right|>(1-\lambda)(b-a)$ and $\omega\left(F ; E^{\lambda}\right)<\infty$.

Proof. Given $\varepsilon>0, x \in[a, b], \lambda, 0<\lambda<1$, there exists $\delta>0, E_{x} \subset$ ]$x-\delta, x+\delta\left[\right.$ such that if $0<h<\delta,\left|E_{x} \cap[x-h, x+h]\right|>2(1-\lambda) h$ and if $u \leqslant x \leqslant v, u, v \in E_{x}$, then $|F(v)-F(u)|<\varepsilon$.

The set of such $E_{x}, a \leqslant x \leqslant b$, defines an AFC, $\Delta$, of $[a, b]$; let $\left\{a_{0}, \ldots, a_{p} ; x_{1}, \ldots, x_{p}\right\}$ be a $\Delta$-partition of $[a, b]$ : and define

$$
E^{\lambda}=\bigcup_{k=1}^{p} E_{x_{k}}
$$

Then $\left|E^{\lambda}\right|>(1-\lambda)(b-a)$ and if $u, v \in E^{\lambda}, u \in\left[a_{m-1}, a_{m}\right], v \in\left[a_{n-1}, a_{n}\right]$, say,

$$
\begin{aligned}
|F(v)-F(u)| \leqslant & \sum_{k=m+1}^{n-1}\left|F\left(a_{k}\right)-F\left(a_{k-1}\right)\right| \\
& +\left|F\left(a_{m}\right)-F(u)\right|+\left|F(v)-F\left(a_{n-1}\right)\right| \leqslant \varepsilon p,
\end{aligned}
$$

which is sufficient to prove the lemma.

THEOREM 4. $F \in A C_{a p}^{*}$ ( $E$ ) if and only if (a) $F \in A C(E)$, (b) for all $\lambda, 0<\lambda<1$, there exists, on each closed contiguous interval $\left[a_{n}, b_{n}\right]$ of $E$, a set $E_{n}^{\lambda}, a_{n}, b_{n} \in E_{n}^{\lambda}$, $\left|E_{n}^{\lambda}\right|>(1-\lambda)\left(b_{n}-a_{n}\right)$ and $\Sigma_{n \in N} \omega\left(F ; E_{n}^{\lambda}\right)<\infty$.

Proof. Let $F \in A C_{a p}^{*}(E), \tilde{E}_{n}^{\lambda}=E_{n}^{\lambda} \cup\left\{a_{n}, b_{n}\right\}$, where $E_{n}^{\lambda}$ are the sets of Definition 1(a)(ii); let $x_{n}, y_{n} \in \tilde{E}_{n}^{\lambda}, n \in N$. Then

$$
\left|F\left(y_{n}\right)-F\left(x_{n}\right)\right| \leqslant\left|F\left(x_{n}\right)-F\left(a_{n}\right)\right|+\left|F\left(y_{n}\right)-F\left(b_{n}\right)\right|+\left|F\left(a_{n}\right)-F\left(b_{n}\right)\right| \text {; }
$$

since $F \in A C(E), \Sigma_{n \in N}\left|F\left(a_{n}\right)-F\left(b_{n}\right)\right|<\infty$ and the result follows from Definition $1(\mathrm{a})(\mathrm{ii})$. The converse is immediate. 
Theorem 5. $F \in A C_{a p}^{*}(E)$ if and only if for all $\varepsilon>0$ there exists $\delta>0$ such that for all $\alpha_{1}<\beta_{1}<\cdots<\beta_{p}$, points of $E$, if $\sum_{k=1}^{p}\left(\beta_{k}-\alpha_{k}\right)<\delta$ then for all $\lambda$, $0<\lambda<1$, there exists $E_{k}^{\lambda} \subset\left[\alpha_{k}, \beta_{k}\right], \alpha_{k}, \beta_{k} \in E_{k}^{\lambda},\left|E_{k}^{\lambda}\right|>(1-\lambda)\left(\beta_{k}-\alpha_{k}\right)$, $1 \leqslant k \leqslant p$, and $\sum_{k=1}^{p} \omega\left(F ; E_{k}^{\lambda}\right)<\varepsilon$.

Proof. (i) Let $F \in A C_{a p}^{*}(E)$; then $F \in A C(E)$ and so given $\varepsilon>0$, there exists $\delta>0$ such that for all $\alpha_{1}<\beta_{1}<\cdots<\beta_{p}$, points of $E$, if $\sum_{k=1}^{P}\left(\beta_{k}-\alpha_{k}\right)<\delta$ then $\sum_{k=1}^{p} V\left(F ; E_{n}\left[\alpha_{k}, \beta_{k}\right]\right)<\varepsilon$. Further, by Theorem 4, and with its notation, there exists $n_{0}$ such that $\Sigma_{n>n_{0}} \omega\left(F ; E_{n}^{\lambda}\right)<\varepsilon$.

Let $\delta_{0}=\min \left\{\delta ; b_{n}-a_{n}, n \leqslant n_{0}\right\}$ and let $\alpha_{1}<\beta_{1}<\cdots<\beta_{p}$, points of $E$, be such that $\sum_{k=1}^{p} \beta_{k}-\alpha_{k}<\delta_{0}$. Define

$$
\tilde{E}_{k}^{\lambda}=E \cap\left[\alpha_{k}, \beta_{k}\right] \cup \bigcup_{n \in N_{k}} E_{n}^{\lambda}
$$

where

$$
n_{k}=\left\{n ;\left[a_{n}, b_{n}\right] \subset\left[\boldsymbol{\alpha}_{k}, \boldsymbol{\beta}_{k}\right]\right\} ;
$$

clearly if $n \in N_{k}$, then $n>n_{0}$. By Lemma 2,

$$
\omega\left(F ; \tilde{E}_{k}\right) \leqslant V\left(F ; E_{n}\left[\alpha_{k}, \beta_{k}\right]\right)+2 \sum_{n \in N_{k}} \omega\left(F ; E_{n}^{\lambda}\right) .
$$

Hence

$$
\sum_{k=1}^{p} \omega\left(F ; \tilde{E}_{k}\right) \leqslant 3 \varepsilon .
$$

(ii) To prove the converse first note that the condition given implies that $F \in A C(E)$. Using the notation of Definition 1(a)(ii) let $N_{0}$ be such that if $n>n_{0}$ then $\Sigma_{n>n_{0}}\left(b_{n}-a_{n}\right)<\delta$ : then from the condition given $E_{n}^{\lambda} \subset\left[a_{n}, b_{n}\right], a_{n}, b_{n} \in$ $E_{n}^{\lambda},\left|E_{n}^{\lambda}\right|>(1-\lambda)\left(b_{n}-a_{n}\right)$ and $\Sigma_{n>n_{0}} \omega\left(F ; E_{n}^{\lambda}\right)<\varepsilon$.

If $n \leqslant n_{0}$ divide each $\left[a_{n}, b_{n}\right]$ into a finite number of intervals each of length less than $\delta$, and we easily see that there exists $E_{n}^{\lambda} \subset\left[a_{n}, b_{n}\right],\left|E_{n}^{\lambda}\right|>(1-\lambda)\left(b_{n}-\right.$ $\left.a_{n}\right)$ and $\omega\left(F ; E_{n}^{\lambda}\right)<\infty$. From this it follows that $F \in A C_{a p}^{*}(E)$.

DEFINITION 6. Let $E$ be a closed set, with closed contiguous integrals $\left[a_{n}, b_{n}\right]$, $n \in N$; let $x \in E^{\prime}, E_{x}$ a set of unit density at $x$ such that there exists $\varepsilon>0$ with $a_{n}, b_{n} \in E_{x}$ if $\left.\left[a_{n}, b_{n}\right] \subset\right] x-\frac{1}{2} \varepsilon, x+\frac{1}{2} \varepsilon$, say if $n \in N_{x}$ for short; we will write for $F:[a, b] \rightarrow \mathbf{R}, a, b$ the extremities of $E$,

$$
\omega_{n, a p}(F)=\sup _{\alpha, \beta \in E_{x} \cap\left[a_{n}, b_{n}\right]}|F(\beta)-F(\alpha)| .
$$

THEOREM 7. If $F \in A C_{a p}^{*}(E)$ then (a) $F \in A C(E)$, (b) for all $x \in E^{\prime}$, $\Sigma_{n \in N_{x}} \omega_{n, a_{p}}(F)<\infty$. 
Proof. It suffices to prove (b). Since $F \in A C_{a p}^{*}(E)$, by Theorem 4 , for all $\lambda$, $0<\lambda<1$, there exists $n_{\lambda}$ such that

$$
\sum_{n>n_{\lambda}} \omega\left(F ; E_{n}^{\lambda}\right) \leqslant \frac{1}{2^{\lambda}}
$$

Let $\varepsilon_{\lambda}=\min _{n \leqslant n_{\lambda}}\left(b_{n}-a_{n}\right), N_{x, \lambda}=\left\{n ;\left[a_{n}, b_{n}\right] \subset\right] x-\frac{1}{2} \varepsilon_{\lambda}, x+\frac{1}{2} \varepsilon_{\lambda}[\}$ when $\Sigma_{n \in N_{x, \lambda}} \cdot \omega\left(F, E_{n}^{\lambda}\right)<1 / 2^{\lambda}$; put $E_{x}^{\lambda}=\bigcup_{\lambda \in N_{x, \lambda}} E_{n}^{\lambda}$. Now define $E_{x}^{0}=\bigcup_{n \geqslant 1} E_{x}^{1 / 2}$, $\varepsilon_{0}=\sup _{n \geqslant 1} \varepsilon_{1 / n}$, when $\left.E_{x}^{0} \subset\right] x-\frac{1}{2} \varepsilon_{0}, x+\frac{1}{2} \varepsilon_{0}\left[;\right.$ let $N_{x}=\left\{n ;\left[a_{n}, b_{n}\right] \subset\right.$ ]$x-\frac{1}{2} \varepsilon_{0}, x+\frac{1}{2} \varepsilon_{0}[\}$ and finally $\left.E_{x}=E_{x}^{0} \cup E \cap\right] x-\frac{1}{2} \varepsilon_{0}, x+\frac{1}{2} \varepsilon_{0}[$.

Then $E_{x}$ has density 1 at $x$ and if $x_{n}, y_{n} \in E_{x} \cap\left[a_{n}, b_{n}\right], n \in N_{x}$,

$$
\sum_{n \in N_{x}}\left|F\left(x_{n}\right)-F\left(y_{n}\right)\right|=\sum_{m \geqslant 1} \sum_{n \in N_{x, 1 / m}}\left|F\left(x_{n}\right)-F\left(y_{n}\right)\right| \leqslant 1 \text {, }
$$

which completes the proof.

THEOREM 8. If $E$ is a closed set with extremities $a, b, a<b, F:[a, b] \rightarrow \mathbf{R}$ and if (a) $F \in C_{a p}[a, b]$, (b) $F \in A C(E)$, (c) for all $x \in E^{\prime}, \Sigma_{n \in N_{x}} \omega_{n, a p}(F)<\infty$, then $F \in A C_{a p}^{*}(E)$.

Proof. If $x \in E^{\prime}$ consider $E_{x} \cap\left[a_{n}, b_{n}\right], n \in N_{x}$, then for all $\lambda, 0<\lambda<1$, there exists $E_{n}^{\lambda} \subset\left[a_{n}, b_{n}\right], a_{n}, b_{n} \in E_{n}^{\lambda}$, such that $\left|E_{n}^{\lambda}\right|>(1-\lambda)\left(b_{n}-a_{n}\right)$ and clearly $\omega\left(F ; E_{n}^{\lambda}\right)<\omega_{n, a p}(F)$. The family of $] x-\frac{1}{2} \varepsilon, x+\frac{1}{2} \varepsilon\left[\operatorname{covers} E^{\prime}\right.$ and so a finite sub-family of these intervals also covers $E^{\prime}$. Hence there exists a finite set of integers $N_{0}$ such that $\Sigma_{n>N_{0}} \omega\left(F ; E_{n}^{\lambda}\right)<\infty$; since $F \in C_{a p}[a, b]$, the intervals $\left[a_{n}, b_{n}\right], n \in N_{0}$, can be handled using Lemma 3 .

Definition 9. If $E$ is a closed set with extremities $a, b, F:[a, b] \rightarrow \mathbf{R}$, then $F$ is $l A C_{a p}^{*}$ on $E, F \in l A C_{a p}^{*}(E)$ if and only if for all $\varepsilon>0$ there exists $\delta>0$ such that for all $\alpha_{1}<\beta_{1}<\cdots<\beta_{p}$, points of $E$, if $\Sigma_{k=1}^{p}\left(\beta_{k}-\alpha_{k}\right)<\delta$, then for all $\lambda$, $0<\lambda<1$, there exists $E_{k}^{\lambda} \subset\left[\alpha_{k}, \beta_{k}\right], \alpha_{k}, \beta_{k} \in E_{k}^{\lambda},\left|E_{k}^{\lambda}\right|>(1-\lambda)\left(\beta_{k}-\alpha_{k}\right)$ such that for all $x_{k} \in E_{k}^{\lambda}, 1 \leqslant k \leqslant p$,

$$
\begin{aligned}
& \sum_{k=1}^{p} F\left(x_{k}\right)-F\left(\alpha_{k}\right)>-\varepsilon, \\
& \sum_{k=1}^{p} F\left(\beta_{k}\right)-F\left(x_{k}\right)>-\varepsilon .
\end{aligned}
$$

REMarks. (1) An analogous definition can be made for $F \in u A c_{a p}^{*}(E)$ and, from Theorem 5, $F \in A C_{a p}^{*}(E)$ if and only if $F \in A C_{a p}^{*}(E) \cap l A C_{a p}^{*}(E)$.

(2) Further, as in Definition 1(b), we can now define the classes $u\left[A C G_{a p}^{*}\right]$ and $l\left[A C G_{a p}^{*}\right]$. 
THEOREM 10. If $F:[a, b] \rightarrow \mathbf{R}, \quad F \in C_{a p}[a, b], l F_{a p}^{\prime}>-\infty$ n.e. then $F \in$ l[ $\left.A C G_{a p}^{*}\right]$.

Proof. Ridder, [19], proves under these conditions that $F \in l[A C G]$; the rest follows from Tolstov's proof of Theorem 1.3(g), Tolstov, [25].

Remark. The basic lemma in Tolstov, [25], can be used to shorten Ridder's result since it shows that certain sets in Ridder's proof are closed.

Corollary 12. If $F:[a, b] \rightarrow \mathbf{R}, F \in C_{a p}[a, b],-\infty<l F_{a p}^{\prime} \leqslant u F_{a p}^{\prime}<\infty$, n.e. then $F \in\left[A C G_{a p}^{*}\right]$.

We can now define a descriptive integral that will be equivalent to the $P_{a p}^{*}$-integral.

Definition 13. If $f:[a, b] \rightarrow \overline{\mathbf{R}}$ then $f \in D_{a p}^{*}, f$ is $D_{a p}^{*}$-integrable, if and only if there exists $F \in C_{a p}[a, b], F \in\left[A C G_{a p}^{*}\right]$ and $F_{a p}^{\prime}=$ fa.e.; then $\int_{a}^{x} f=F(x)-F(a)$.

REMARK. The basic properties of the class of approximately continuous $-[A C G]$ functions, of which the approximately continuous $-\left[A C G_{a p}^{*}\right]$ functions is a sub-class, Ridder, [18, 19], Kubota, [9], show that this definition is meaningful.

THEOREM 14. If $f \in P_{a p}^{*}$ then $f \in D_{a p}^{*}$, with integrals equal.

Proof. This follows from Theorems 1.3(b), (g), and the remark following Definition 1.

To prove the converse of Theorem 14 we will use the $R_{a p}^{*}$-integral and for this need to show that this integral has what are usually called Cauchy and Harnack properties. That the $R_{a p}^{*}$-integral has the Cauchy property follows from the fact that the equivalent $P_{a p}^{*}$-integral does, Theorem 1.3(f), but we will give an independent proof.

Theorem 15. If $f \in R_{a p}^{*}[\alpha, \beta]$, for all $\beta, \beta, a<\alpha<\beta<b$ and if

$$
\lim _{\substack{\alpha \rightarrow a \\ \beta \rightarrow b}} \int_{\alpha}^{\beta} f
$$

exists, with value I say, then $f \in R_{a p}^{*}[a, b]$, and $\int_{a}^{b} f=I$. 
Proof. It is sufficient to consider the case where for all $\beta, a<\beta<b$, $f \in R^{*}[a, \beta]$ and $\lim _{\beta \rightarrow b} \int_{p}^{\beta} \int_{a}^{\beta} f=I$. Let $a=\beta_{0}<\beta_{1}<\cdots, \lim _{n \rightarrow \infty} \beta_{n}=b, \varepsilon>0$, then since $k \geqslant 1, f \in R_{a p}^{*}\left[\beta_{k}, \beta_{k-1}\right]$, there exists AFC, $\Delta_{k}$, of $\left[\beta_{k}, \beta_{k-1}\right]$ such that for all $\Delta_{k}$-partitions of $\left[\beta_{k}, \beta_{k-1}\right],\left\{a_{0}^{k}, \ldots, ; x_{1}^{k} \cdots\right\}$,

$$
\left|\int_{\beta_{k-1}}^{\beta_{k}} f-\sum \cdot f\left(x_{i}^{k}\right)\left(a_{i}^{k}-a_{i-1}^{k}\right)\right|<\frac{\varepsilon}{2^{k}} .
$$

Since $\lim _{\beta \rightarrow b} \int_{p}^{\beta} f=I$, given $\varepsilon>0$ there exists $\delta>0$ and a set $A$ of density 1 at $b$, $A \subset[b-\delta, b]$, such that if $x \in A,\left|I-\int_{a}^{x} f\right|<\varepsilon$, and $|(b-x) f(b)|<\varepsilon . \Delta=$ $\cup_{x \in A}[x, b] \cup \cup_{k \geqslant 1} \Delta_{k}$ is an AFC of $[a, b]$ and consider the $\Delta$-partition $\left\{a_{0}, \ldots, a_{n} ; x_{1}, \ldots, x_{n}\right\}$ of $[a, b]$ :

$$
\begin{aligned}
&\left|I-\sum_{i=1}^{n} f\left(x_{i}\right)\left(a_{i}-a_{i-1}\right)\right| \leqslant\left|\int_{a}^{a_{n-1}} f-\sum_{i=1}^{n-1} f\left(x_{i}\right)\left(a_{i}-a_{i-1}\right)\right| \\
&+\left|I-\int_{a}^{a_{n-1}} f\right|+\left|f(b)\left(b-a_{n-1}\right)\right| \\
&<3 \varepsilon,
\end{aligned}
$$

and so $\int_{a}^{b} f$ exists, with value $I$.

THEOREM 16. Let $E$ be a perfect set, end points $a, b$, with closed contiguous intervals in $[a, b],\left[a_{n}, b_{n}\right], n \in N$; suppose that $f 1_{E} \in R_{a p}^{*}[a, b]$ and that for all $n \in N, f \in R_{a p}^{*}\left[a_{n}, b_{n}\right]$; suppose further that for all $x \in E$ there exists a set $E_{x}$ of unit density at $x, \delta>0$, with $a_{n}, b_{n} \in E_{x}$ if $\left.\left[a_{n}, b_{n}\right] \subset\right] x-\frac{1}{2} \delta, x+\frac{1}{2} \delta\left[, n \in N_{x}\right.$, for short and $\Sigma_{n \in N_{x}}\left\{\sup _{\alpha, \beta \in E_{x} \cap\left[a_{n}, b_{n}\right]}\left|\int_{\alpha}^{\beta} f\right|\right\}<\infty$; then $f \in R_{a p}^{*}[a, b]$ and

$$
\int_{a}^{b} f=\int_{a}^{b} f 1_{E}+\sum_{n \in N} \int_{a_{n}}^{b_{n}} f .
$$

Proof. It is sufficient to prove that $f\left(1-1_{E}\right) \in R_{a p}^{*}[a, b]$. Note that the above conditions imply that for all $\varepsilon>0$ there exists $n_{0}$ such that

$$
\sum_{n>n_{0}}\left\{\sup _{\alpha, \beta \in E_{x} \cap\left[a_{n}, b_{n}\right]}\left|\int_{\alpha}^{\beta} f\right|\right\}<\varepsilon,
$$

and so, in particular, the right-hand side of (1) is defined.

For each $n \in N$ there exists AFC $\Delta_{n}$ of $\left[a_{n}, b_{n}\right]$ such that for all $\Delta_{n}$-partitions of $\left[a_{n}, b_{n}\right],\left\{a_{0}^{n}, \ldots ; x_{1}^{n} \cdots\right\}$,

$$
\left|\int_{a_{n}}^{b_{n}} f-\sum f\left(x_{i}^{n}\right)\left(a_{i}^{n}-a_{n-1}^{n}\right)\right|<\frac{\varepsilon}{2^{n}} .
$$

At each $x \in E$ there exists $\tilde{E}_{x} \subset E_{x}$, of density 1 at $x$, containing all $a_{n}, b_{n}$, $n>n_{0}$, and $\left.\left[a_{n}, b_{n}\right] \subset\right] x-\frac{1}{2} \delta, x+\frac{1}{2} \delta\left[:\right.$ let $\tilde{E}_{x}^{*}=\left\{[u, v] ; u \leqslant x \leqslant v, u_{1} v \in \tilde{E}_{x}\right\}$. 
Consider $\Delta=\cup_{n \in N} \Delta_{n} \cup \cup_{x \in E} \tilde{E}_{x}$, an $\mathrm{AFC}$ on $[a, b]$, and $\left\{a_{0}, \ldots, a_{p}\right.$; $\left.x_{1}, \ldots, x_{p}\right\}$ any $\Delta$-partition of $[a, b]$ :

$$
\begin{aligned}
& \sum_{n \in N} \mid \int_{a_{n}}^{b_{n}} f-\sum_{i=1}^{p} f\left(1-1_{E}\right)\left(x_{i}\right)\left(a_{i}-a_{i-1}\right) \mid \\
& \leqslant \sum_{n>n_{0}}\left|\int f\right|+\left|\sum_{n \leqslant n_{0}} \int_{a_{n}}^{b_{n}} f-\sum f\left(x_{i}^{n}\right)\left(a_{i}^{n}-a_{i-1}^{n}\right)\right| \\
&<2 \varepsilon,
\end{aligned}
$$

which completes the proof.

THEOREM 17. If $f \in D_{a p}^{*}$ then $f \in R_{a p}^{*}$ and the integrals are equal.

Proof. Let $f \in D_{a p}^{*}, E=\left\{x ; a \leqslant x \leqslant b\right.$ and $f$ is not $R_{a p}^{*}$-integrable in some neighbourhood of $x\}$; assume $E \neq \varnothing$. From Theorem $15, E$ is perfect and if $\left[a_{n}, b_{n}\right], n \in N$ are the closed continuous intervals of $E$, in $[a, b]$, then $f \in$ $R_{a p}^{*}\left[a_{n}, b_{n}\right], n \in N$. If $F(x)=D_{a p}^{*}-\int_{a}^{x} f$ then $f \in\left[A C G_{a p}^{*}\right]$ and so $E$ contains a portion $E_{0}$ on which $F$ is $A C_{a p}^{*}$; let $\alpha, \beta$ be the extremities of $E_{0}$. Since $F \in A C\left(E_{0}\right), F_{a p}^{\prime}=f$ a.e. on $E_{0}$ and $f$ is $L$-integrable there, and so $f 1_{E} \in R_{a p}^{*}[a, b]$. Further since $F \in A C_{a p}^{*}\left(E_{0}\right)$, by Theorem 8, all the conditions of Theorem 16 are satisfied on $[\alpha, \beta]$, and so $f \in R_{a p}^{*}[\alpha, \beta]$. This proves that $E=\varnothing$, and so $f \in R_{a p}^{*}[a, b]$.

COROLlaRY 18. $P_{a p}^{*}=R_{a p}^{*}=D_{a p}^{*}$.

\section{An approximate total}

The approximate-total* of $f, f:[a, b] \rightarrow \mathbf{R}, T_{a p}^{*}-\int_{a}^{b} f$, is constructed by the transfinite induction as indicated below; if the construction is possible we say that $f \in T_{a p}^{*}$.

The process uses four operations:

(1) if $a \leqslant \alpha \leqslant \beta \leqslant b, f \in L[\alpha, \beta]$ then $T_{a p}^{*}-\int_{\alpha}^{\beta} f=L-\int_{\alpha}^{\beta} f$;

(2) if for all $\alpha^{\prime}, \beta^{\prime}, a \leqslant \alpha<\alpha^{\prime}<\beta^{\prime}<\beta \leqslant b$ we have evaluated $T_{a p}^{*}-\int_{\alpha^{\prime}}^{\beta^{\prime}} f$ and if

$$
\lim _{\substack{\alpha^{\prime} \rightarrow \alpha p \\ \beta^{\prime} \rightarrow \boldsymbol{\beta}}} T_{a p}^{*}-\int_{\alpha^{\prime}}^{\beta^{\prime}} f
$$

exists, then $T_{a p}^{*}-\int_{\alpha}^{\beta} f$ is defined to be this limit;

(3) if $T^{*}-\int_{\alpha}^{\beta} f$ and $T^{*}=\int_{\beta}^{\delta} f, a \leqslant \alpha<\beta<\beta \leqslant b$, have been evaluated then $T_{a p}^{*}-\int_{\alpha}^{\delta} f$ is defined to be their sum; 
(4) if $P \subset[a, b]$ is perfect, with extremities $\alpha, \beta$, and if $f 1_{P} \in L[\alpha, \beta]$, and if $f \in T_{a p}^{*}\left[\alpha_{n}, \beta_{n}\right],\left[\alpha_{n}, \beta_{n}\right]$ being the closed contiguous intervals of $P$ in $[\alpha, \beta]$, $n \in N$, and if further for all $x \in P$ there exists a set $E_{x}$ of density 1 at $x, \delta>0$ with $a_{n}, b_{n} \in E_{x}$, if $\left.\left[a_{n}, b_{n}\right] \subset\right] x-\frac{1}{2} \delta, x+\frac{1}{2} \delta\left[, n \in N_{x}\right.$, for short, and $\sum_{n \in N_{x}}\left\{\sup _{\alpha_{n}^{\prime}, \beta_{n}^{\prime} E_{x} \cap\left[\alpha_{n}, \beta_{n}\right]}\left|T_{a p}^{*}-\int_{\alpha_{n}^{\prime \prime}}^{\beta_{n}^{\prime}} f\right|\right\}<\infty$, then $T_{a p}^{*}-\int_{\alpha}^{\beta} f$ is evaluated as $L-$ $\int_{\alpha}^{\beta} f 1_{P}+\sum_{n \in N} T_{a p}^{*}-\int_{\alpha_{n}}^{\beta_{n}} f$.

REMARK. This operation is related to that used in an integral defined by Kubota, [11, 12], in the same way as the corresponding operation in the special Denjoy integral is related to that in the general Denjoy integral; Saks, [20; page 255].

The construction of $T_{a p}^{*}-\int_{a}^{b} f$ can now be described as follows.

Stage 1: Step 1. Let $E=\{x ; a \leqslant x \leqslant b, f$ is not summable at $x\}$. If $E$ is not nowhere dense, $f \notin T_{a p}^{*}$, if $E$ is nowhere dense proceed to

Step 2. For all $[\alpha, \beta],[\alpha, \beta] \cap E=\varnothing$ compute $T_{a p}^{*}-\int_{\alpha}^{\beta} f$ by operation (1).

Step 3. If $[\alpha, \beta]$ is a closed contiguous interval of $E$ see if

$$
\lim _{\substack{\alpha^{\prime} \rightarrow a p \\ \beta \rightarrow \beta}} T_{a p}^{*}-\int_{\alpha}^{\beta} f
$$

exists; if not $f \notin T_{a p}^{*}$, if so compute $T_{a p}^{*}-\int_{\alpha}^{\beta} f$ by operation (2).

Step 4. For all $[\alpha, \beta],[\alpha, \beta] \cap E^{\prime}=\varnothing$ compute $T_{a p}^{*}-\int_{\alpha}^{\beta} f$ by operation (3).

Step 5. Applying step 3 to the contiguous intervals of $E^{\prime}$, then by a transfinite process using steps 4 and 3 , we either find that $f \notin T_{a p}^{*}$, or will have computed $T_{a p}^{*}-\int_{\alpha}^{\beta} f$ for all $[\alpha, \beta]$, closed contiguous intervals of the perfect kernel $P$ of $E$; if $P=\varnothing$ we have completed the calculation, if not proceed to

Stage 2: Step 1. Let $\tilde{E}=\left\{x ; x \in P\right.$ and $f 1_{p}$ is not summable at $\left.x\right\}$. If $\tilde{E}$ is not nowhere dense in $P, f \notin T_{a p}^{*}$; if $\tilde{E}$ is nowhere dense on $P$, proceed to

Step 2. For all $[\alpha, \beta],[\alpha, \beta] \cap \tilde{E}=\varnothing$ compute $T_{a p}^{*}-\int_{\alpha}^{\beta} f$ as described in stage 3 below. If this is not possible $f \notin T_{a p}^{*}$, if it is use steps 3,4 of stage 1 to compute, if possible $T_{a p}^{*}-\int_{\alpha}^{\beta} f$ for all $[\alpha, \beta]$, closed contiguous intervals of the perfect kernel of $\tilde{E}$.

Step 3. A transfinite process using the above steps then either finds $f \notin T_{a p}^{*}$ or computes $T_{a p}^{*}-\int_{\alpha}^{\beta} f$ on the closed contiguous intervals of $E_{1}=E, E_{2}=\tilde{E}$, $E_{3}, \ldots, E_{\lambda}, \ldots$, where if $\lambda$ has a predecessor $E_{\lambda}$ is nowhere dense in the perfect kernel, $P_{\lambda-1}$ of $E_{\lambda-1}$ and $E_{\lambda}=\left\{x ; x \in P_{\lambda-1}\right.$ and $f 1_{P_{\lambda-1}}$ is not summable at $\left.x\right\}$, while if $\lambda$ has no predecessor $E_{\lambda}=\bigcap_{\mu<\lambda} E_{\mu}$. For some $\nu<\Omega, E_{\nu}=\varnothing, E_{\nu-1} \neq \varnothing$, that is, either $P_{\nu-1}=\varnothing$ or $f 1_{P_{\nu-1}} \in L[a, b]$; in either case stages 1-3 applied to $E_{\nu-1}$ completes the computation. 
Stage 3: (From step 2 of stage 2 we have to compute $T_{a p}^{*}-\int_{\alpha}^{\beta} f$ where $[\alpha, \beta]$ defines a closed portion of a perfect set $P, Q$ say, with $f 1_{Q}$ summable, and on the closed contiguous intervals in $[\alpha, \beta]$ of $Q,\left[\boldsymbol{\alpha}_{n}, \beta_{n}\right], T_{a p}^{*}-\int_{\boldsymbol{\alpha}_{n}}^{\beta_{n}} f$ has already been computed, $n \in N$.)

Step 1. Let $x$ be a regular point of $Q$ if there exists a set $E_{x}$, of density 1 at $x$, $\delta>0$, with $\alpha_{n}, \beta_{n} \in E_{x}$ if $\left.\left[\alpha_{n}, \beta_{n}\right] \subset\right] x-\frac{1}{2} \delta, x+\frac{1}{2} \delta\left[, n \in N_{x}\right.$ for short, and $\sum_{n \in N_{x} s}\left\{\sup _{\alpha_{n}^{\prime}, \beta_{n}^{\prime} \in E_{x} \cap\left\{\alpha_{n}, \beta_{n}\right]}\left|T_{a p}^{*}-\int_{\alpha^{\prime}}^{\beta^{\prime}} f\right|\right\}<\infty$; let $E$ be the set of non-regular points of $Q$. If $E$ is not nowhere dense in $Q, f \notin T_{a p}^{*}$, if it is proceed to

Step 2. For all $\left[\alpha^{\prime}, \beta^{\prime}\right],\left[\alpha^{\prime}, \beta^{\prime}\right] \cap E=\varnothing$ compute $T_{a p}^{*}-\int_{\alpha^{\prime}}^{\beta^{\prime}} f$ by operation (4).

Step 3. Proceed as in stage 1 to obtain $T_{a p}^{*}-\int_{\alpha^{\prime}}^{\beta^{\prime}} f$ on all $\left[\alpha^{\prime}, \beta^{\prime}\right]$ closed contiguous intervals of the perfect kernel of $E$; then proceed to stage 2 again.

To facilitate the discussion of the $T_{a p}^{*}$-integral we define for all $\alpha, 0 \leqslant \alpha \leqslant \Omega$, on $[a, b]$ an integral $L_{a p}^{*, \alpha}$; this follows the ideas of Saks, [20], and Kubota, $[11,12]$.

(a) $L_{a p}^{*, 0}=L$.

(b) If for all $\alpha<\beta \leqslant \Omega$ we have defined $L_{a p}^{*, \alpha}$ in such a way that the integrals are compatible and if $\alpha<\alpha^{\prime}<\beta$ then $L_{a p}^{*, \alpha} \subset l_{a p}^{*, \alpha^{\prime}}$ then $I_{1}^{\beta}$ is the integral defined by

$$
I_{1}^{\beta}=\bigcup_{\alpha<\beta} L_{a p}^{*, \alpha}, \quad I_{1}^{\beta}-\int_{a}^{b} f=L_{a p}^{*, \alpha_{0}}-\int_{a}^{b} f,
$$

where

$$
\alpha_{0}=\min \left\{\alpha ; f \in L_{a p}^{*, \alpha}\right\} .
$$

(c) (i) If $\beta<\Omega$ then $I_{2}^{\beta}$ is the integral $\left(I_{1}^{\beta}\right)_{a p}^{C}$, see Definition 1(a) below; and

$$
L_{a p}^{*, \beta}=\left(I_{2}^{\beta}\right)_{a p}^{H^{*}},
$$

see Definition 1(b) below;

(ii) if $\beta=\Omega$,

$$
L_{a p}^{* \Omega}=I_{1}^{\Omega} .
$$

Definition 1. If $I$ is an integral let $S_{f}=S=\{x ; f$ is not $I$-integrable at $x\}$; then:

(a) the approximate Cauchy extension of $I, I_{a p}^{C}$, is defined as follows: $f \in I_{a p}^{C}$ if and only if there exists $F \in C_{a p}$ such that if $\left[a^{\prime}, b^{\prime}\right] \cap S=\phi$ then $I-\int_{a^{\prime}}^{b^{\prime}} f=$ $F\left(b^{\prime}\right)-F\left(a^{\prime}\right)$ then $I_{a p}^{C}-\int_{a}^{b} f=F(b)-F(a)$.

(b) the approximate Harnack* extension of $I, I_{a p}^{H^{*}}$, is defined as follows: $f \in I_{a p}^{H^{*}}$ if and only if (i) $f 1_{s} \in L$, (ii) if $\left[a_{n}, b_{n}\right], n \in N$ are the closed contiguous intervals of $S$ in $[a, b]$ then $f$ is $I$-integrable on each, and if $x$ is a limit point of the 
$\left[a_{n}, b_{n}\right]$ there exists a set $E_{x}$ of unit density, $\delta>0$, with $a_{n}, b_{n} \in E_{x}$ if $\left[a_{n}, b_{n}\right] \subset$ ]$x-\frac{1}{2} \delta, x+\frac{1}{2} \delta\left[, x \in N_{x}\right.$ for short, and $\Sigma_{n \in N_{x}}\left\{\sup _{a_{n}^{\prime}, b_{n}^{\prime} \in E_{x} \cap\left[a_{n}, b_{n}\right]}\left|I-\int_{a_{n}^{\prime}}^{b_{n}^{\prime}} f\right|\right\}$ $<\infty$, then

$$
I_{a p}^{H^{*}}-\int_{a}^{b} f=L-\int_{a}^{b} f 1_{S}+\Sigma_{n \in N} I-\int_{a}^{b} f
$$

The following theorem is then easily deduced, using the methods of Saks, [20], and Kubota, $[11,12]$.

THEOREM 2. (a) $\left(\left(L_{a p}^{*, \Omega}\right)_{a p}^{C}\right)_{a p}^{H^{*}}=L_{a p}^{*, \Omega}$.

(b) $L_{a p}^{*, \Omega}=T_{a p}^{*}$.

(c) $L_{a p}^{*, \Omega}=D_{a p}^{*}$.

(d) If $I$ is an approximately continuous integral such that (i) $L \subset I$, (ii) $\left(I_{a p}^{C}\right)_{a p}^{*}=I$, then $D_{a p}^{*} \subset I$.

COROLLARY 3. $P_{a p}^{*}=R_{a p}^{*}=D_{a p}^{*}=T_{a p}^{*}=L_{a p}^{*, \Omega}$.

\section{References}

[1] A. Aleksandrov, 'Über die Äquivalenz des Perronschen und des Denjoyschen Integralbegriffes', Math. Z. 20 (1924), 213-222.

[2] L. S. Bosanquet, 'A property of Cesàro-Perron integrals', Proc. Edinburgh Math. Soc. (2) 6 (1940), 160-165.

[3] P. S. Bullen, 'Non-absolute integrals; a survey', Real Anal. Exchange 5 (1980), 195-259.

[4] J. C. Burkill, 'The approximately continuous Perron integral', Math. Z. 34 (1931), 270-278.

[5] M. E. Grimshaw, 'The Cauchy property of the generalised Perron integrals', Proc. Cambridge Philos. Soc. 30 (1934), 15-18.

[6] R. Henstock, Theory of integration (London, 1963).

[7] R. Henstock, Linear analysis (London, 1967).

[8] R. Henstock, 'The variation on the real line', Proc. Roy. Irish Acad. Sect. A 79 (1979), 1-10.

[9] Y. Kubota, 'An integral of Denjoy', I, Proc. Japan. Acad. 40 (1964), 713-717.

[10] Y. Kubota, 'On the compatibility of the $A P$-and the $D$-integrals', Proc. Japan Acad. 44 (1968), 330-333.

[11] Y. Kubota, 'A characterization of the approximately continuous Denjoy integral', Canad. $J$. Math. 22 (1970), 219-226.

[12] Y. Kubota, 'A constructive definition of the approximately continuous Denjoy integral', Canad. Math. Bull. 15 (1972), 103-108.

[13] Y. Kubota, 'A direct proof that the $R C$-integral is equivalent to the $D^{*}$-integral', Proc. Amer. Math. Soc. 80 (1980), 293-296.

[14] Y. Kubota, 'An elementary theory of the special Denjoy integral', Math. Japon. 24 (1980), 507-520.

[15] R. O’Malley, 'Selective derivatives', Acta Math. Acad. Sci. Hungar. 29 (1977), 77-79.

[16] W. F. Pfeffer, The Riemann-Stieltjes approach to integration (Technical Report, TWISK 187, National Research Inst. for Math. Sci., Pretoria, 1980). 
[17] J. Ridder, 'Über den Perronschen Integralbegriff und seine Beziehung zu den $R$-, $L$ - und D-Integralen', Math. Z. 34 (1932), 234-269.

[18] J. Ridder, 'Über approximativ stetige Denjoy-Integrale', Fund. Math. 21 (1933), 1-10.

[19] J. Ridder, 'Über die Gegenseitigen Beziehungen verschiedener approximativ stetiger DenjoyPerron Integrale', Fund. Math. 22 (1934), 136-162.

[20] S. Saks, Theory of the integral, 2nd ed., revised (New York, 1937).

[21] D. W. Solomon, Denjoy integration in abstract spaces (Mem. Amer. Math. Soc. 85, 1969).

[22] G. Sunouchi and M. Utagawa, 'The generalized Perron integrals', Tôhoku Math. J. 1 (1949), 95-99.

[23] B. S. Thomson, 'On full coverings', Real Anal. Exchange 6 (1980-81), 77-93.

[24] G. P. Tolstov, 'Sur quelques propriétés des fonctions approximativement continues', Mat. Sb. 5 (1939), 637-645.

[25] G. P. Tolstov, 'Sur l'intégrale de Perron', Mat. Sb. 5 (1939), 647-659.

[26] G. P. Tolstov, 'La méthode de Perron pour l'intégrale de Denjoy', Mat. Sb. 8 (1940), 149-167.

[27] Z. Zahorski, 'Ueber die Menge der Punkte in welchen die Ableitung unendlich ist', Tôhoku Math. J. 48 (1941), 321-332.

\section{Mathematics Department}

The University of British Columbia

Vancouver, British Columbia

Canada 